\title{
Magnetic order and spin dynamics in $\mathrm{La}_{2} \mathrm{O}_{2} \mathrm{Fe}_{2} \mathrm{OSe}_{2}$ probed by ${ }^{57} \mathrm{Fe}$ Mössbauer, ${ }^{139} \mathrm{La} \mathrm{NMR}$, and muon-spin relaxation spectroscopy
}

\author{
M. Günther, ${ }^{1, *}$ S. Kamusella, ${ }^{1}$ R. Sarkar, ${ }^{1}$ T. Goltz, ${ }^{1}$ H. Luetkens, ${ }^{2}$ G. Pascua, ${ }^{2}$ S.-H. Do, ${ }^{3}$ K.-Y. Choi, ${ }^{3}$ H. D. Zhou,,${ }^{4,5}$ \\ C. G. F. Blum, ${ }^{6}$ S. Wurmehl, ${ }^{6,1}$ B. Büchner, ${ }^{6,1}$ and H.-H. Klauss ${ }^{1}$ \\ ${ }^{1}$ Institut für Festkörperphysik, Technische Universität Dresden, D-01069 Dresden, Germany \\ ${ }^{2}$ Paul Scherrer Institut, 5232 Villigen PSI, Switzerland \\ ${ }^{3}$ Department of Physics, Chung-Ang University, Seoul 156-756, Republic of Korea \\ ${ }^{4}$ Department of Physics and Astronomy, University of Tennessee, Knoxville, Tennessee 37996-1200, USA \\ ${ }^{5}$ National High Magnetic Field Laboratory, Florida State University, Tallahassee, Florida 32306-4005, USA \\ ${ }^{6}$ Leibniz-Institut für Festkörper- und Werkstoffforschung (IFW) Dresden, D-01171 Dresden, Germany \\ (Received 1 April 2014; revised manuscript received 15 October 2014; published 7 November 2014)
}

\begin{abstract}
We present a detailed local probe study of the magnetic order in the oxychalcogenide $\mathrm{La}_{2} \mathrm{O}_{2} \mathrm{Fe}_{2} \mathrm{OSe}_{2}$ utilizing ${ }^{57} \mathrm{Fe}$ Mössbauer, ${ }^{139} \mathrm{La} \mathrm{NMR}$, and muon-spin relaxation spectroscopy. This system can be regarded as an insulating reference system of the Fe arsenide and chalcogenide superconductors. From the combination of the local probe techniques we identify a noncollinear magnetic structure similar to $\mathrm{Sr}_{2} \mathrm{~F}_{2} \mathrm{Fe}_{2} \mathrm{OS}_{2}$. The analysis of the magnetic order parameter yields an ordering temperature $T_{\mathrm{N}}=90.1 \mathrm{~K}$ and a critical exponent of $\beta=0.133$, which is close to the two-dimensional Ising universality class as reported in the related oxychalcogenide family.
\end{abstract}

DOI: 10.1103/PhysRevB.90.184408

PACS number(s): 74.70.Xa, 76.80.+y, 76.60.-k, 76.75.+i

\section{INTRODUCTION}

One of the important issues to understand the iron-based superconductors is the role of electron correlations. The antiferromagnetically ordered state of the parent compounds of $R \mathrm{FeAsO}$ ( $R=$ rare earth) [1,2] and $\mathrm{BaFe}_{2} \mathrm{As}_{2}$ [3] pnictide superconductors can be understood either as a spin density wave order of an itinerant multiband Fermi surface [4], or due to a frustrated $J_{1}-J_{2}$ interaction of localized spins [5,6]. Strongly localized systems with layered transition metal ( $\mathrm{Tm}$ ) pnictide or (oxy-) chalcogenide structures serve as reference systems to understand the properties of the ironbased superconductors. Moreover, due to the interplay of three different intraplane exchange interactions of $T m$ ions, the oxychalcogenide systems show a rich variety of magnetically ordered ground states. $G$-type antiferromagnetic (AFM) order with magnetic Mn moments aligned along the crystallographic $c$ axis is reported for $\mathrm{La}_{2} \mathrm{O}_{2} \mathrm{Mn}_{2} \mathrm{OSe}_{2}$ [7]. A noncollinear arrangement of magnetic moments parallel to the $a-b$ plane is concluded for $\mathrm{Sr}_{2} \mathrm{~F}_{2} \mathrm{Fe}_{2} \mathrm{OS}_{2}$ [Fig. 9(e)] and a different one for $\mathrm{La}_{2} \mathrm{O}_{2} \mathrm{Co}_{2} \mathrm{OSe}_{2}$ [with a possible magnetic structure according to the model in Fig. 9(d)] [8,9].

The $R_{2} \mathrm{O}_{2} T m_{2} \mathrm{OCh}$ and $A_{2} \mathrm{~F}_{2} T m_{2} \mathrm{OCh}_{2}$ oxychalcogenides exist in a rich variety of compositions: systems with $R=\mathrm{La}, \mathrm{Ce}, \mathrm{Pr}, \mathrm{Nd}, \mathrm{Sm} ; A=\mathrm{Sr}, \mathrm{Ba} ; \mathrm{Tm}=\mathrm{Fe}, \mathrm{Co}, \mathrm{Mn}$; and $C h=\mathrm{Se}, \mathrm{S}$ are reported [7,10-15]. Among these isostructural materials with $I 4 / \mathrm{mmm}$ symmetry, all reported systems with $T m=$ Fe exhibit a transition to antiferromagnetic order near $T_{\mathrm{N}}=100 \mathrm{~K}$. Nonmetallic and antiferromagnetic properties in $\mathrm{La}_{2} \mathrm{O}_{2} \mathrm{Fe}_{2} \mathrm{OCh}_{2}$ and $\mathrm{Sr}_{2} \mathrm{~F}_{2} \mathrm{Fe}_{2} \mathrm{OS}_{2}$ are consistent with theoretical studies indicating Mott-insulating behavior [8,16,17]. ${ }^{57} \mathrm{Fe}$ Mössbauer studies on $\mathrm{Nd}_{2} \mathrm{O}_{2} \mathrm{Fe}_{2} \mathrm{OSe}_{2}$ and $\mathrm{Sr}_{2} \mathrm{~F}_{2} \mathrm{Fe}_{2} \mathrm{OS}_{2}$ found critical exponents close to the two-dimensional (2D) Ising universality class, indicating anisotropical, dominantly 2D magnetic exchange interactions $[8,11,12]$.

*guenth42@googlemail.com
In these systems, within a $\mathrm{Fe}_{2} \mathrm{OCh}$ layer, three different magnetic exchange interactions of $\mathrm{Fe}^{2+}$ ions are considered: a diagonal nearest neighbor (nn) interaction $J_{\mathrm{nn}}$ and two next nearest neighbor (nnn) interactions $J_{\mathrm{nnn} 1}$ and $J_{\mathrm{nnn} 2}$ via $\mathrm{O}^{2-}$ or two $C h^{2-}$ ions, respectively [Fig. 9(b)] [11]. The nature of the magnetic interactions and possible models of magnetic order in the iron based oxychalcogenides had been studied extensively $[8,11,12,16-18]$.

The magnetic ground state of $\mathrm{La}_{2} \mathrm{O}_{2} \mathrm{Fe}_{2} \mathrm{OSe}_{2}$ was proposed to obey a plaquette AFM order for on-site Hubbard interaction $U=1.5 \mathrm{eV}$ or $U=3 \mathrm{eV}$, and the Neel state for $U=$ $4.5 \mathrm{eV}$ [16]. From this the deduced interactions are reported to be $\mathrm{FM}$ for the $\mathrm{Fe}-\mathrm{Se}_{2}-\mathrm{Fe}$ pathway, AFM for the $\mathrm{Fe}-\mathrm{O}-\mathrm{Fe}$ interaction, and AFM for the nearest neighbor coupling $J_{\mathrm{nn}}$. The transition from plaquette AFM order $(p>1)$ to the Néel state $(p<1)$ is driven by the parameter $p=J_{\mathrm{nnn} 1} /\left|J_{\mathrm{nn}}\right|$. While in the plaquette state two nn Fe magnetic moments are aligned parallel and two antiparallel, with stronger $J_{\text {nn }}$ in the Néel state all AFM nn interactions are satisfied, but not the AFM $J_{\mathrm{nnn} 1}$ mediated by oxygen ions.

A neutron diffraction study on $\mathrm{La}_{2} \mathrm{O}_{2} \mathrm{Fe}_{2} \mathrm{OSe}_{2}$ concluded a collinear and columnar AFM order below $T_{\mathrm{N}}=90 \mathrm{~K}$ [18]. In this model none of the three interactions is satisfied for all nn and nnn Fe moments. For one axis of the basal plane the $\mathrm{Fe}$ magnetic moments are aligned parallel along $\mathrm{Fe}-\mathrm{O}-\mathrm{Fe}$ and $\mathrm{Fe}_{-} \mathrm{Se}_{2}-\mathrm{Fe}$ chains and antiparallel along the other. Again two nn Fe magnetic moments are aligned parallel and two antiparallel.

In contrast, Fuwa et al. introduced two different noncollinear models of magnetic order for $\mathrm{Nd}_{2} \mathrm{O}_{2} \mathrm{Fe}_{2} \mathrm{OSe}_{2}$ [12]. These models are consistent with an axial anisotropy of magnetic $\mathrm{Fe}$ moments, aligned along perpendicular O$\mathrm{Fe}-\mathrm{O}$ chains in the $\mathrm{Fe}_{2} \mathrm{OSe}_{2}$ layer. One of these models was found to describe noncollinear magnetic order in $\mathrm{Sr}_{2} \mathrm{~F}_{2} \mathrm{Fe}_{2} \mathrm{OS}_{2}$ [8]. In Figs. 9(c)-9(e) the three magnetic structures consistent with AFM $J_{\mathrm{nnn} 1}$ interaction and an orientation of magnetic moments along O-Fe-O chains are shown. 
Recently it was shown that a collinear and columnar AFM order and the noncollinear order concluded for $\mathrm{Sr}_{2} \mathrm{~F}_{2} \mathrm{Fe}_{2} \mathrm{OS}_{2}$ are indistinguishable in elastic neutron scattering on $\mathrm{La}_{2} \mathrm{O}_{2} \mathrm{Fe}_{2} \mathrm{OSe}_{2}$ powder, whereas inelastic experiments are only consistent with the noncollinear model [19]. Moreover, gapped $(\approx 5 \mathrm{meV})$ and confined (below $\approx 25 \mathrm{meV}$ ) magnetic excitation was reported.

In this study we present ${ }^{57} \mathrm{Fe}$ Mössbauer, ${ }^{139} \mathrm{La} \mathrm{NMR}$, and muon-spin relaxation ( $\mu \mathrm{SR})$ experiments on polycrystalline $\mathrm{La}_{2} \mathrm{O}_{2} \mathrm{Fe}_{2} \mathrm{OSe}_{2}$. We find long-range magnetic order below $T_{\mathrm{N}}=90.1 \mathrm{~K}$ and deduce a critical exponent $\beta=0.133$. The magnetic order is deduced to obey a noncollinear structure according to a ferromagnetic (FM) interaction $J_{\text {nnn2 }}$ [Fig. 9(e)]. We first give a brief introduction to the experimental setup in Sec. II. In Sec. III we present our results of ${ }^{57} \mathrm{Fe}$ Mössbauer spectroscopy. ${ }^{139}$ La NMR field-swept and $\mu$ SR spectra are presented in Secs. IV and V. Finally we give a summary in Sec. VI.

\section{EXPERIMENTAL DETAILS}

Polycrystalline samples of $\mathrm{La}_{2} \mathrm{O}_{2} \mathrm{Fe}_{2} \mathrm{OSe}_{2}$ were prepared by the solid-state reaction method using stoichiometric amounts of $\mathrm{La}_{2} \mathrm{O}_{3}, \mathrm{Fe}$, and $\mathrm{Se}$. The resulting powder were ground in an argon glove box, pressed into a pellet, and placed inside a quartz ampoule under vacuum. The pellet was heated by following the routine described in Ref. [18]. The single phase of $\mathrm{La}_{2} \mathrm{O}_{2} \mathrm{Fe}_{2} \mathrm{OSe}_{2}$ was confirmed by $\mathrm{x}$-ray powder diffraction experiments performed on a STOE Stadi $\mathrm{P}$ powder diffractometer with $\operatorname{Mo} K_{\alpha 1}$ radiation $(\lambda=0.70926 \AA)$ at $293 \mathrm{~K}$. The diffractometer is equipped with a curved $\mathrm{Ge}(111)$ monochromator and a $6^{\circ}$-linear position sensitive detector. The structural analysis was carried out by Rietveld refinement utilizing the FULLPROF software package [20,21]; see also Fig. 1. The refined structural parameters are presented in Table I and are in good agreement with earlier studies [10,13].

All experimental work presented here were done on one single batch of crushed polycrystalline material.

For ${ }^{57} \mathrm{Fe}$ Mössbauer spectroscopy a thin absorber of $2.6 \mathrm{mg} / \mathrm{cm}^{2} \mathrm{Fe}$ area density was prepared by evaporation of a methanol suspension of the powdered sample. A Wissel

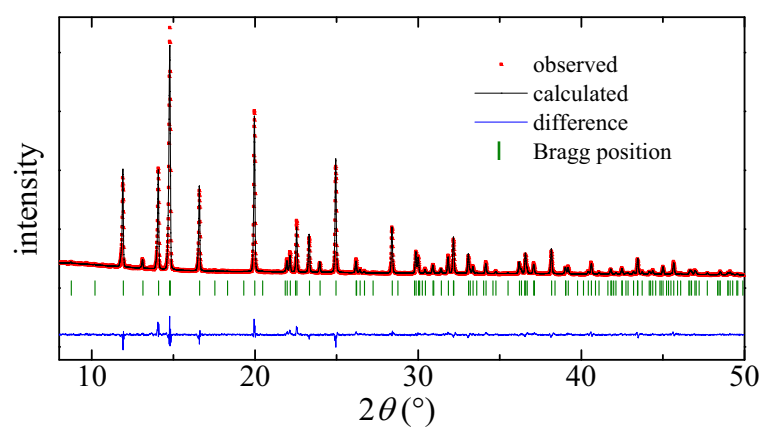

FIG. 1. (Color online) Room temperature powder x-ray diffraction spectrum and analysis on $\mathrm{La}_{2} \mathrm{O}_{2} \mathrm{Fe}_{2} \mathrm{OSe}_{2}$. Red symbols show the observed intensity. The black solid line represents the refined spectrum. Vertical green markers show the positions of the calculated Bragg reflections. The lower blue line is the difference between observed and calculated intensities.
TABLE I. Summary of crystallographic parameters from Rietveld refined XRD experiment.

\begin{tabular}{lccccc}
\hline \hline & $\mathrm{La}$ & $\mathrm{Fe}$ & $\mathrm{Se}$ & $\mathrm{O}(1)$ & $\mathrm{O}(2)$ \\
\hline Wykoff position & $4 c$ & $4 c$ & $4 e$ & $4 d$ & $2 b$ \\
$x(a)$ & $1 / 2$ & $1 / 2$ & 0 & $1 / 2$ & $1 / 2$ \\
$y(a)$ & $1 / 2$ & 0 & 0 & 0 & $1 / 2$ \\
$z(c)$ & $0.1845(1)$ & 0 & $0.0966(1)$ & $1 / 4$ & 0 \\
\hline \multicolumn{5}{c}{ space group $I 4 / m m m, a=4.08744(4) \AA, c=18.6061(3) \AA}$, \\
\multicolumn{5}{c}{$R_{\mathrm{p}}=2.76 \%, R_{\mathrm{wp}}=3.75 \%, \chi^{2}=2.39$} \\
\hline \hline
\end{tabular}

spectrometer was operated in sinusoidal mode and photons were detected with a KeTek-SDD detector.

${ }^{139}$ La NMR experiments in a temperature range of $5.0 \leqslant$ $T \leqslant 170 \mathrm{~K}$ were performed by conventional pulsed NMR techniques with the duration of a $\pi / 2$ pulse equal to $6 \mu \mathrm{s}$. The NMR data were recorded in a $8 \mathrm{~T}$ superconducting magnet system with a ${ }^{4} \mathrm{He}$ variable temperature insert, using a Tecmag LapNMR spectrometer. The polycrystalline powder was placed in a glass tube inside a $\mathrm{Cu}$ coil with a frequency of the resonant circuits of $44.0 \mathrm{MHz}$ and $25.5 \mathrm{MHz}$. The spinlattice relaxation rate was measured by using the saturation recovery method.

An extensive search was carried out to resolve the ${ }^{77} \mathrm{Se}$ signal, however without success. Since the crystallographic Se site is located much closer to the Fe than the La site, a strong magnetic hyperfine coupling can lead to a nuclear relaxation too fast to be measured.

Zero magnetic field $\mu$ SR spectra were recorded using the GPS instrument at the PSI Villigen, Switzerland, in a temperature range $5 \leqslant T \leqslant 160 \mathrm{~K}$. The data were analyzed using the free software package MUSRFIT [22].

\section{III. ${ }^{57} \mathrm{Fe}$ MÖSSBAUER SPECTROSCOPY}

${ }^{57} \mathrm{Fe}$ Mössbauer spectroscopy allows to study microscopically the strength and alignment of ordered Fe magnetic moments with respect to the electric field gradient (EFG) principal axis within the distorted $\mathrm{FeO}_{2} \mathrm{Se}_{4}$ octahedra. Moreover it is sensitive to temperature dependent changes of the electronic surrounding via the electric quadrupole interaction.

Figure 2 shows the ${ }^{57} \mathrm{Fe}$ Mössbauer spectra at selected temperatures. In the paramagnetic regime down to $T=120 \mathrm{~K}$ we find a single asymmetric doublet with a quadrupole splitting of $\Delta v_{\mathrm{QS}}=1.98 \mathrm{~mm} / \mathrm{s}$ (Fig. 3). This corresponds to a principal EFG component of $V_{z z}=119 \mathrm{~V} / \AA^{2}$ and is close to the values found in earlier studies $[12,15]$. The asymmetric absorption intensity of the doublet is interpreted in terms of texture caused by the flaky shape of the crystallites. These flakes are suspected to be oriented flat on the bottom of the sample holder, resulting in a preferred orientation of the $c$ axis parallel to the $\gamma$ beam. With decreasing temperature below $T_{\mathrm{N}}$ a sextet is developed, indicating long-range magnetic ordering. Due to the $D_{2 h}$ symmetry of the ${ }^{57} \mathrm{Fe}$ site an asymmetric electric field gradient, with asymmetry $\eta>0$, is expected. The analysis of the spectra is done by diagonalization of the full static 


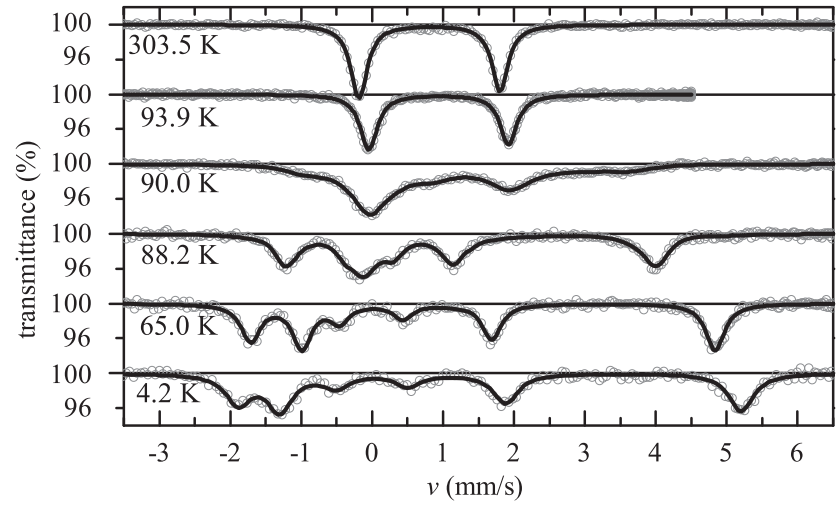

FIG. 2. Temperature dependence of ${ }^{57} \mathrm{Fe}$ Mössbauer spectra (open circles). Solid lines represent fits as described in the text. The paramagnetic spectra consist of an asymmetric doublet due to texture effects. A single sextet indicates full long-range magnetic order rapidly arising below $T_{\mathrm{N}}$.

hyperfine Hamiltonian

$$
\begin{aligned}
H_{\mathrm{s}}= & \frac{e Q V_{z z}}{4 I(2 I-1)}\left[\left(3 I_{z}^{2}-I^{2}\right)+\frac{\eta}{2}\left(I_{+}^{2}+I_{-}^{2}\right)\right] \\
& -g_{I} \mu_{\mathrm{N}} B_{\mathrm{hyp}}\left(\frac{I_{+} e^{-i \phi}+I_{-} e^{+i \phi}}{2} \sin \theta+I_{z} \cos \theta\right),
\end{aligned}
$$

with nuclear spin operators $I_{z}, I_{+}=I_{x}+i I_{y}$, and $I_{-}=$ $I_{x}-i I_{y} . B_{\text {hyp }}$ is the hyperfine field at the ${ }^{57} \mathrm{Fe}$ site. $Q, g_{I}$, and $\mu_{\mathrm{N}}$ denote the nuclear quadrupole moment, $g$ factor, and magneton. The polar angle $\phi$ and the azimuthal angle $\theta$ describe the orientation of the Fe hyperfine field $B_{\text {hyp }}$ with respect to the EFG $z$ axis. Due to the preferred orientation of the sample flakes parallel to the bottom of the sample holder, i.e., the preferred orientation of the $\gamma$ beam parallel to the $c$ axis, a texture function

$$
D\left(\phi_{\gamma}, \theta_{\gamma}\right)=\sin ^{N} \phi_{\gamma}\left|\cos ^{N} \theta_{\gamma}\right|=\cos ^{N} \epsilon
$$

is introduced to account for corrections of the line intensities with respect to the geometrical setup. Here the polar angle $\phi_{\gamma}$ and the azimuthal angle $\theta_{\gamma}$ describe the orientation of the $\gamma$

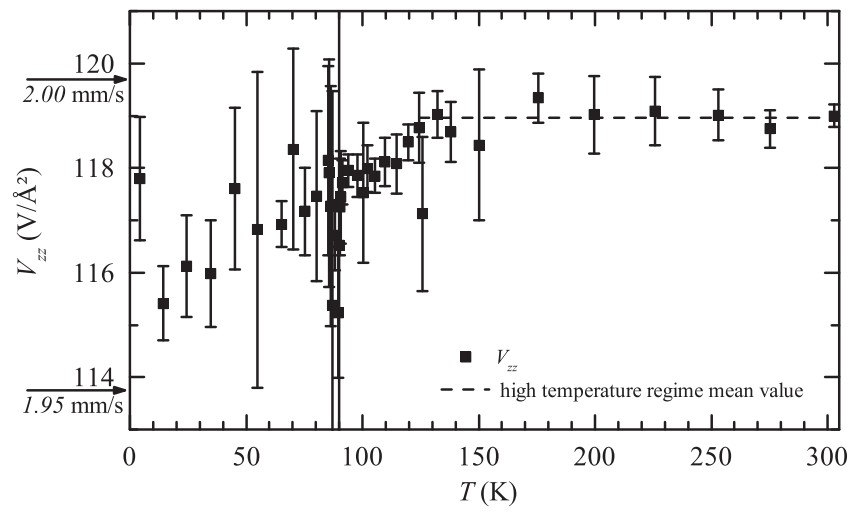

FIG. 3. Temperature dependence of the principal axis component $V_{z z}$ of the EFG. The dashed line indicates the mean value for $T>$ $120 \mathrm{~K}$. The anomaly of $V_{z z}(T)$ occurs at the same temperature as that of $c$ axis thermal expansion [18].
TABLE II. Comparison of ${ }^{57} \mathrm{Fe}$ Mössbauer studies on different oxychalcogenide systems with $\mathrm{Tm}=\mathrm{Fe}$. The ordering temperature $T_{\mathrm{N}}$, low-temperature ${ }^{57} \mathrm{Fe}$ saturation hyperfine field $B_{\text {sat }}$, and the critical exponent $\beta$ are found to vary only in small ranges. " $*$ " denotes results of the present work.

\begin{tabular}{lccc}
\hline \hline & $T_{\mathrm{N}}(\mathrm{K})$ & $B_{\text {sat }}(\mathrm{T})$ & \multicolumn{1}{c}{$\beta$} \\
\hline $\mathrm{La}_{2} \mathrm{O}_{2} \mathrm{Fe}_{2} \mathrm{OSe}_{2} *$ & 90.1 & 20.3 & 0.133 \\
$\mathrm{La}_{2} \mathrm{O}_{2} \mathrm{Fe}_{2} \mathrm{OSe}_{2}[12]$ & & 21 & \\
$\mathrm{Nd}_{2} \mathrm{O}_{2} \mathrm{Fe}_{2} \mathrm{OSe}_{2}[12]$ & 88.3 & 20 & 0.104 \\
$\mathrm{Sr}_{2} \mathrm{~F}_{2} \mathrm{Fe}_{2} \mathrm{OS}_{2}[11]$ & 106.2 & 20.65 & 0.15 \\
$\mathrm{Ba}_{2} \mathrm{~F}_{2} \mathrm{Fe}_{2} \mathrm{OSe}_{2}[11]$ & 83.6 & 20.4 & 0.118 \\
\hline \hline
\end{tabular}

beam with respect to the EFG $z$ axis. Additionally the equivalent description by the angle $\epsilon$ between the crystallographic $c$ axis and the $\gamma$ beam is given. With this the preferred orientation can be smoothly tuned by the model parameter $N$.

In the AFM ordered state we find constant $N=1.14$ and $\eta=0.15$ (3) within error bars. While a simple point charge model calculation estimates $\eta<0.146$ in $\mathrm{La}_{2} \mathrm{O}_{2} \mathrm{Fe}_{2} \mathrm{OSe}_{2}$, a smaller value was reported for $\mathrm{Nd}_{2} \mathrm{O}_{2} \mathrm{Fe}_{2} \mathrm{OSe}_{2}(\eta \approx 0.1)$ [12]. $N$ and $\eta$ are fixed for further analysis of $V_{z z}$ and $B_{\text {hyp }}$. While decreasing the temperature the magnetic volume fraction increases rapidly. At $88 \mathrm{~K}$ the sample is $100 \%$ magnetic. At the lowest measured temperature $T=4.2 \mathrm{~K}$ the spectrum is described by a Zeeman splitting with maximum hyperfine field $B_{\text {sat }}=20.3(1)$ T. All spectra are fitted with a single Fe site. The isomer shift of $0.82 \mathrm{~mm} / \mathrm{s}$ at room temperature is typical for a high spin state of $\mathrm{Fe}^{2+}[23]$. The absolute value of the ${ }^{57} \mathrm{Fe}$ hyperfine field at low temperatures $B_{\text {sat }}$ is close to that of other oxychalcogenide systems (Table II). For $\mathrm{Sr}_{2} \mathrm{~F}_{2} \mathrm{Fe}_{2} \mathrm{OS}_{2}$ a saturation field $B_{\text {sat }}=20.65 \mathrm{~T}$ and the low-temperature ordered $\mathrm{Fe}$ magnetic moment $m_{\mathrm{Fe}}=3.3(1) \mu_{\mathrm{B}}$ are reported [8,11]. Taking into account the very similar local coordination of the $\mathrm{Fe}$ ions in $\mathrm{La}_{2} \mathrm{O}_{2} \mathrm{Fe}_{2} \mathrm{OSe}_{2}$ and $\mathrm{Sr}_{2} \mathrm{~F}_{2} \mathrm{Fe}_{2} \mathrm{OS}_{2}$, the same conversion factor $A=B_{\text {sat }} / m_{\mathrm{Fe}}=6.26 \mathrm{~T} / \mu_{\mathrm{B}}$ between the ${ }^{57} \mathrm{Fe}$ hyperfine field and the ordered magnetic moment, as calculated from the $\mathrm{Sr}_{2} \mathrm{~F}_{2} \mathrm{Fe}_{2} \mathrm{OS}_{2}$ values, is valid for both materials. From this a low-temperature ordered $\mathrm{Fe}$ magnetic moment of $m_{\mathrm{Fe}}=B_{\text {sat }} / A=3.2 \mu_{\mathrm{B}}$ in $\mathrm{La}_{2} \mathrm{O}_{2} \mathrm{Fe}_{2} \mathrm{OSe}_{2}$ is deduced from the saturation field.

Mössbauer spectroscopy clearly shows that $\theta=0$ in the magnetically ordered regime, i.e., the magnetic hyperfine field $\mathbf{B}_{\text {hyp }}$ is oriented parallel to the $z$ axis of the EFG principal axis system (Fig. 4). Considering that the EFG strongest component is aligned along the $\mathrm{O}-\mathrm{Fe}-\mathrm{O}$ chains (also calculated in a LSDA $+U$ approach) [12], this proves that the ordered Fe magnetic moments are oriented parallel to $\mathrm{O}-\mathrm{Fe}-\mathrm{O}$ chains, resulting in a noncollinear magnetic order. This was already concluded for the $\mathrm{Nd}$ system [12]. Considering AFM $J_{\mathrm{nnn} 1}$ intraplane exchange interactions, three different models of noncollinear order are possible (see Fig. 9).

Fitting the sublattice magnetization for temperatures above $0.6 T_{\mathrm{N}}, M(T) \propto B_{\text {hyp }}(T) \propto\left(1-T / T_{\mathrm{N}}\right)^{\beta}$ yields a transition temperature $T_{\mathrm{N}}=90.1 \pm 0.1 \mathrm{~K}$. The critical exponent $\beta=$ 0.133 is close to that of the magnetic square planar Ising model $(\beta=1 / 8)$.

Figure 3 shows the temperature dependence of the EFG component $V_{z z}(T) . V_{z z}(T)$ is constant at high temperatures 


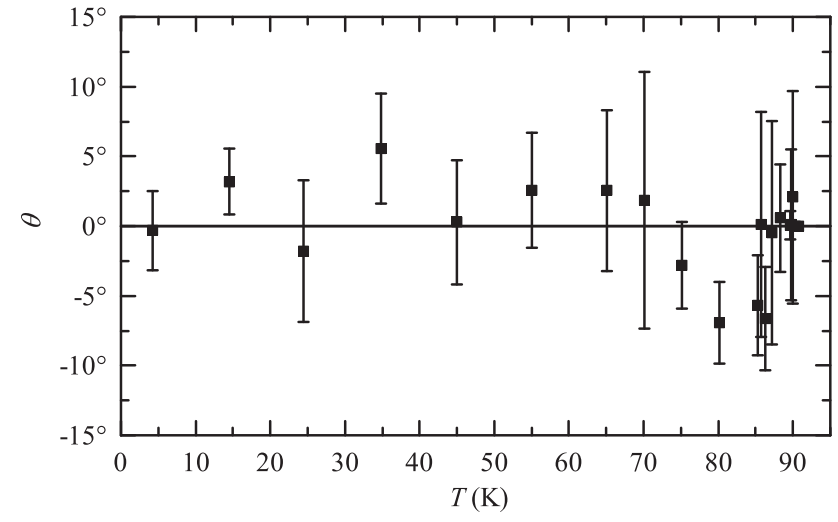

FIG. 4. The angle $\theta$ between the principal EFG $z$ axis and the ${ }^{57} \mathrm{Fe}$ hyperfine field $\mathbf{B}_{\text {hyp }}$ in the AFM ordered state obtained from a fit to Mössbauer spectra.

above $120 \mathrm{~K}$ and then decreases gradually below. This onset temperature is in accordance with an anomaly of the thermal expansion along the $c$ axis, detected in neutron scattering experiments [18]. The Debye-Waller factor as well as the quadratic Doppler shift can be described in the Debye model (Fig. 5). For both datasets a Debye temperature $\Theta_{\mathrm{D}}=241 \mathrm{~K}$ is obtained and no anomaly is found below $120 \mathrm{~K}$.

\section{IV. ${ }^{139}$ La NMR}

In this section we present field-swept ${ }^{139} \mathrm{La}$ NMR spectra and spin-lattice relaxation data. Figure 6 shows ${ }^{139} \mathrm{La}$ spectra at different temperatures. At high temperatures a typical nuclear spin $I=7 / 2$ powder line shape is observed. For $T>T_{\mathrm{N}}$ the spectra consist of a split central transition and three pairs of satellites. Note that the most outer satellite at the high-field side was not observed at $44.0 \mathrm{MHz}$, but has been verified in measurements at a frequency of $25.5 \mathrm{MHz}$. In the paramagnetic state the deduced quadrupole frequency is $v=3.1 \mathrm{MHz}$ and the asymmetry parameter $\eta=0$. Due to the local $C_{4 v}$ symmetry of $\mathrm{La}$, a vanishing $\eta$ is reasonable and the EFG principal $z$ axis is assumed to match the crystallographic $c$ axis. These values are fixed for the analysis of the magnetically ordered regime $T<T_{\mathrm{N}}$, where two effects lead to more complex spectra: (1) A significant broadening of the central transition and the satellites is attributed to static hyperfine

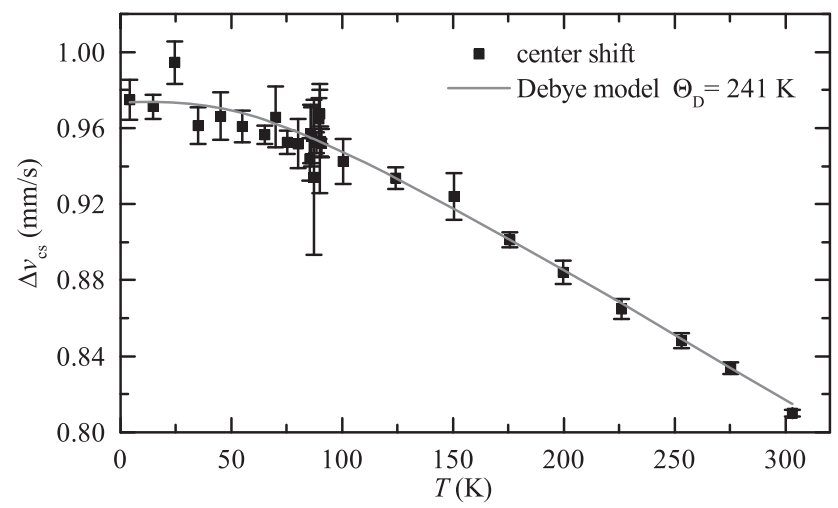

FIG. 5. Temperature dependence of the center shift $\Delta v_{\mathrm{cs}}$.

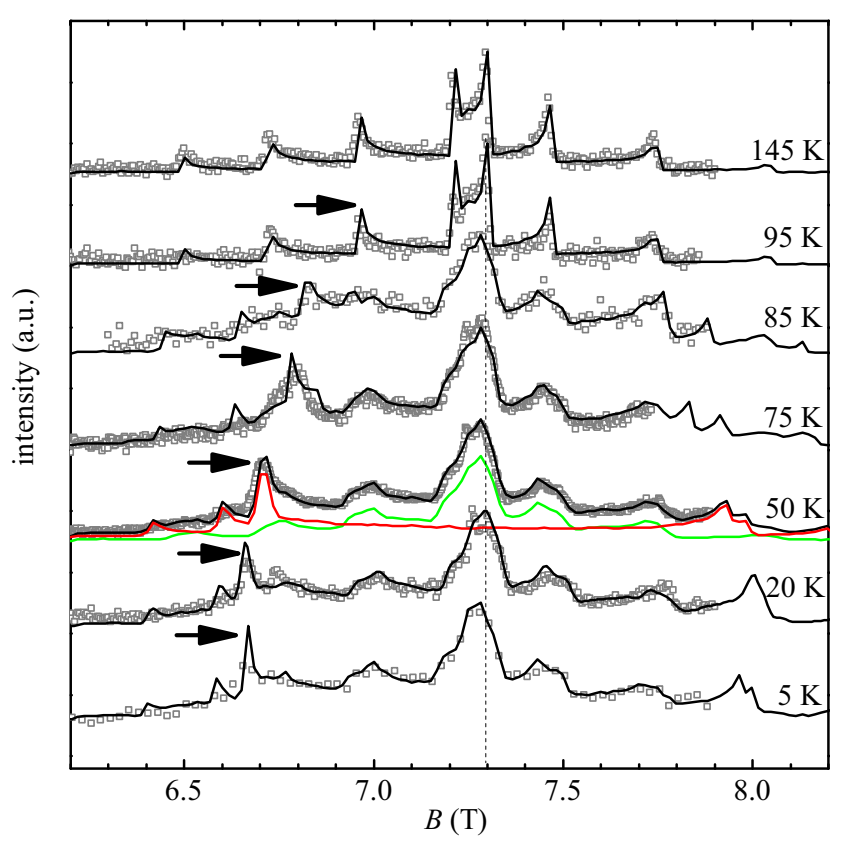

FIG. 6. (Color online) Temperature dependence of the ${ }^{139} \mathrm{La}$ field-swept NMR spectra at $44.0 \mathrm{MHz}(20 \mathrm{~K}$ spectrum at 44.1 $\mathrm{MHz}$ ). For $T>T_{\mathrm{N}}$ typical nonmagnetic $I=7 / 2$ powder spectra are observed. Black arrows indicate the position of ${ }^{139} \mathrm{La}$ signal in the ordered regime stemming from a satellite position at high temperatures. Black solid lines are simulated spectra. Green and red solid lines at $50 \mathrm{~K}$ represent calculated sub spectra due to magnetically nonequivalent sites (see text). The vertical dash-dotted line indicates the field where ${ }^{139}\left(1 / T_{1}\right)$ were measured.

fields. (2) Additional ${ }^{139} \mathrm{La}$ intensity appears at $85 \mathrm{~K}$, near 6.8 T, and strongly shifts to lower fields when cooling the sample. Since there is only one crystallographic La position in the unit cell, the NMR experiments show two magnetically nonequivalent sites.

To analyze the field-swept spectra, the nuclear Hamiltonian

$$
H=-\hbar \gamma\left(\mathbf{B}+\mathbf{B}_{\|}+\mathbf{B}_{\perp}\right) \mathbf{I}+h \frac{v}{6}\left[3 I_{z}^{2}-\mathbf{I}^{2}+\eta\left(I_{x}^{2}-I_{y}^{2}\right)\right]
$$

was diagonalized for 25000 random orientations of the external field $\mathbf{B}$, with respect to the EFG principal $z$ axis, at each sweep step $(\gamma / 2 \pi=6.0146 \mathrm{MHz} / \mathrm{T}) . \mathbf{B}_{\|}$and $\mathbf{B}_{\perp}$ are hyperfine fields parallel and perpendicular to the $a-b$ plane. Transitions of nuclear sublevels corresponding to $44.0 \mathrm{MHz}$ (in a window of $70 \mathrm{kHz}$ corresponding to the signals FWHM) provide the intensity for a given field point.

While the modeled spectra yield an accurate description of the experimental data without any additional internal field $\left(\mathbf{B}_{\|}=0 \mathrm{mT}\right.$ and $\left.\mathbf{B}_{\perp}=0 \mathrm{mT}\right)$ at temperatures $T>T_{\mathrm{N}}$, two local fields $\mathbf{B}_{1}$ and $\mathbf{B}_{2}$ at magnetically nonequivalent ${ }^{139} \mathrm{La}$ sites must be introduced to describe the observed spectra in the ordered state.

Site 1: Choosing $\mathbf{B}_{1}$ parallel to the $a-b$ plane results in a broadening of the simulated spectra. The green lines in Figs. 6 and 7 are simulations with $\left|\mathbf{B}_{1}\right|=B_{1, \|}=35 \mathrm{mT}$ and reproduce some features of NMR line broadening at $T=50 \mathrm{~K}$. Any 


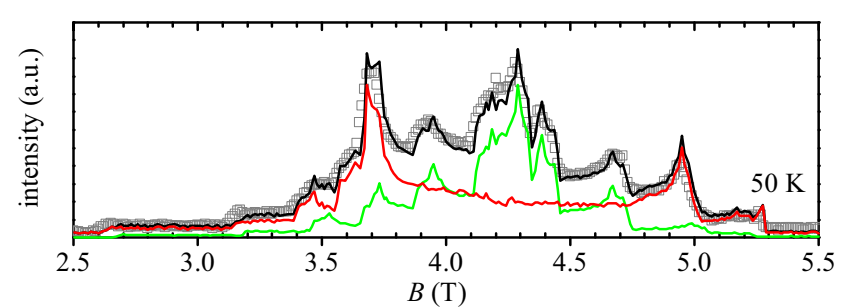

FIG. 7. (Color online) Gray squares show ${ }^{139} \mathrm{La}$ field-swept NMR spectrum at $25.5 \mathrm{MHz}$ and $50 \mathrm{~K}$. Green and red solid lines represent calculated spectra due to the two magnetically nonequivalent sites (see text). The total simulated spectrum is shown as a black line.

additional component parallel to the $c$-axis will impair the simulation and we find $B_{1, \perp}=0 \mathrm{mT}$.

Site 2: To describe the second signal, a simulation with $\mathbf{B}_{2} \| \mathbf{c}$ is shown as a red line. Here $B_{2, \perp}=1040 \mathrm{mT}$ at $50 \mathrm{~K}$. Additional components of the hyperfine field parallel to the $a-b$ plane, in the order of $35 \mathrm{mT}$, will not interfere the simulation.

Black lines are spectral sums of equal weighting for both ${ }^{139} \mathrm{La}$ sites and model our field-swept data very well in the magnetically ordered regime. The temperature dependency of $B_{2, \perp}$ is shown in Fig. 8. Additional field-swept spectra at a resonance frequency of $25.5 \mathrm{MHz}$ yields similar results and confirm the magnetic origin of line broadening and shifting (Fig. 7).

We calculated the hyperfine coupling constants at the ${ }^{139} \mathrm{La}$ sites, using localized Fe magnetic dipole moments for three noncollinear models of magnetic order (Fig. 9). For each model (c) and (d), equivalent values of hyperfine coupling parallel and perpendicular to the $c$ axis are obtained at all ${ }^{139}$ La sites. Therefore those models cannot describe our NMR field swept spectra in the magnetically ordered state. Only model (e) results in magnetically nonequivalent ${ }^{139} \mathrm{La}$ sites:

Site 1: For site 1 the small hyperfine coupling is parallel to the $a-b$ plane. The value of the components reads $A_{1, \|}=$ $8.3 \mathrm{mT} / \mu_{\mathrm{B}}$ and $A_{1, \perp}=0 \mathrm{mT} / \mu_{\mathrm{B}}$. The parallel component

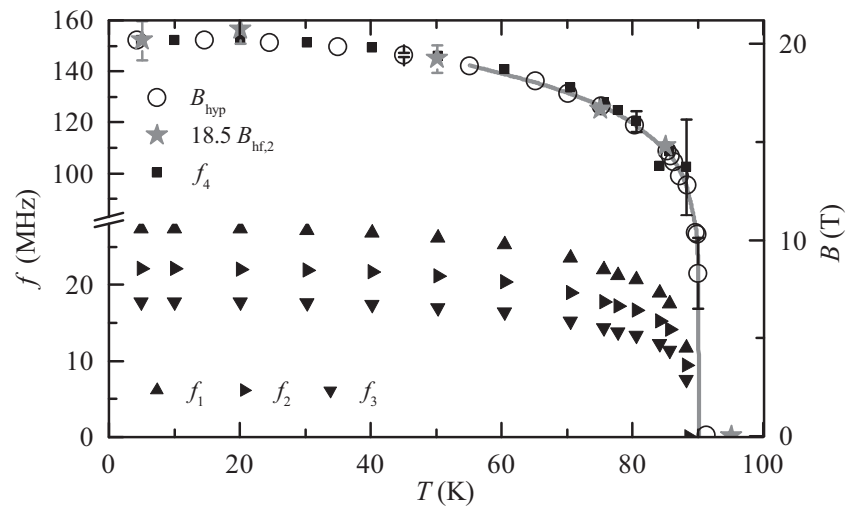

FIG. 8. Magnetic order parameter determined from Mössbauer, $\mathrm{NMR}$, and $\mu$ SR measurements. Open circles represent ${ }^{57} \mathrm{Fe}$ hyperfine fields fitted to Mössbauer spectra. Gray stars refer to ${ }^{139} \mathrm{La} \mathrm{hyperfine}$ fields $B_{2, \perp}$ scaled by a factor of 18.5 . Black solid symbols show fitted $\mu \mathrm{SR}$ frequencies $f_{1}-f_{4}$ as described in the text. Representative error bars are shown. The gray line shows the fit of sublattice magnetization of the Mössbauer data according to the text. (a)
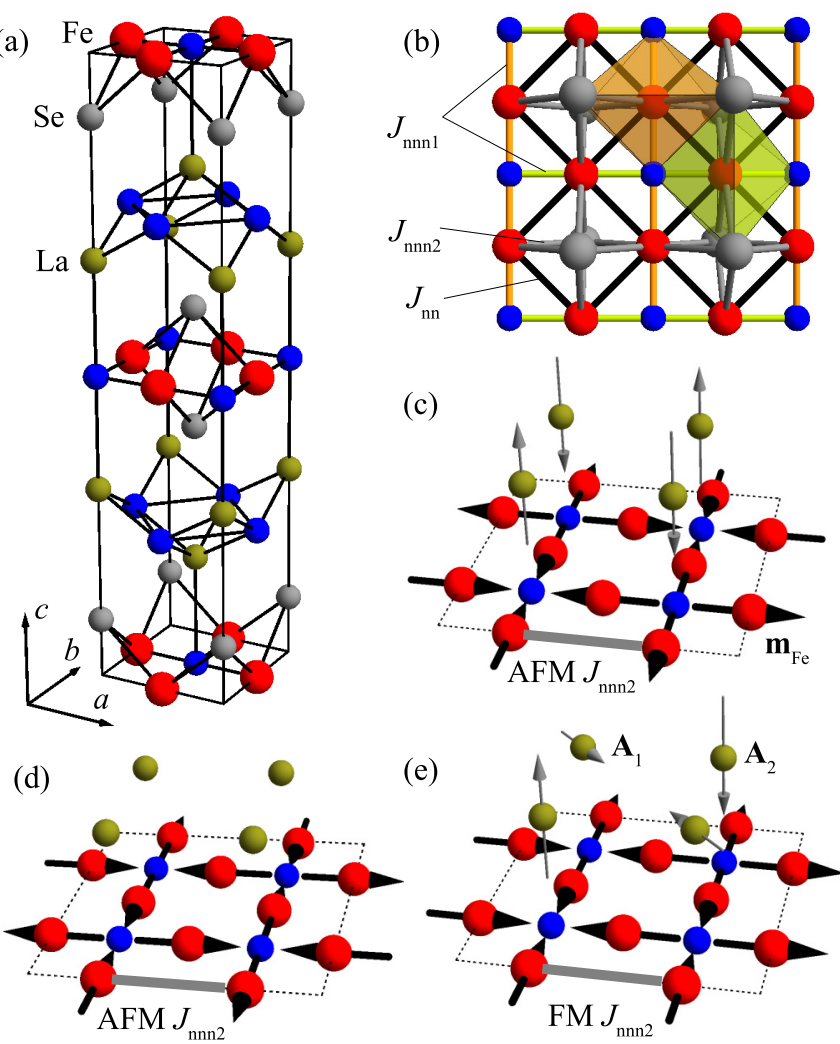

(e)

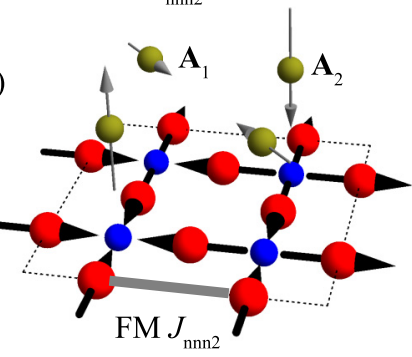

FIG. 9. (Color online) (a) Crystallographic unit cell of $\mathrm{La}_{2} \mathrm{O}_{2} \mathrm{Fe}_{2} \mathrm{OSe}_{2}$. (b) Magnetic exchange interactions in the $\mathrm{Fe}_{2} \mathrm{OSe}_{2}$ layer. Green and orange distorted octahedra sketch the coordination of Fe ions along perpendicular Fe-O-Fe chains. Green and orange lines symbolize the $J_{\mathrm{nnn} 1}$ superexchange pathway along these chains. Gray and black lines represent $J_{\mathrm{nnn} 2}$ and $J_{\mathrm{nn}}$. For AFM $J_{\mathrm{nnn} 2}$ and moments aligned along $\mathrm{O}-\mathrm{Fe}-\mathrm{O}$ chains there are three realizations of magnetic structure as discussed in the literature. (c)-(e) Sketches of the $\mathrm{Fe}_{2} \mathrm{O}$ plane for the three different noncollinear models of magnetic structure. Additionally representative La sites are shown. The gray arrows indicate the calculated dipolar hyperfine coupling. (c) AFM $J_{\text {nnn2 }}$ yields strong hyperfine fields at all ${ }^{139}$ La sites. (d) AFM $J_{\mathrm{nnn} 2}$ yields no hyperfine fields at all ${ }^{139}$ La sites. (e) Ferromagnetic $J_{\mathrm{nnn} 2}$ interaction results in the model of magnetic order as described for $\mathrm{Sr}_{2} \mathrm{~F}_{2} \mathrm{Fe}_{2} \mathrm{OS}_{2}$ and yields magnetically nonequivalent ${ }^{139} \mathrm{La}$ sites.

primarily arises due to the nnn $\mathrm{FeO}_{2}$ plane, while all contributions from the nn plane cancel with respect to the symmetry.

Site 2: For site 2 a strong hyperfine coupling is essentially parallel to the $c$ axis. The values of the components are $A_{2, \|}=$ $8.3 \mathrm{mT} / \mu_{\mathrm{B}}$ and $A_{2, \perp}=81.5 \mathrm{mT} / \mu_{\mathrm{B}}$. The strong component parallel to the $c$ axis primarily arises due to the coupling to $\mathrm{Fe}$ magnetic dipolar moments located in the $\mathrm{nn} \mathrm{FeO}_{2}$ plane. Again the parallel component is dominantly due to the nnn $\mathrm{FeO}_{2}$ plane.

This model is identified to be consistent with our experimental results.

Comparing the hyperfine fields deduced from the NMR field-swept experiment to the ordered magnetic $\mathrm{Fe}$ moment deduced from Mössbauer spectroscopy, we obtain a hyperfine coupling parallel to the $a-b$ plane of $A_{1, \|}^{\prime}=11.4 \mathrm{mT} / \mu_{\mathrm{B}}$ for site 1 and $A_{2, \perp}^{\prime}=340 \mathrm{mT} / \mu_{\mathrm{B}}$ for site 2. The dipolar model yields a good qualitative description of our field- 


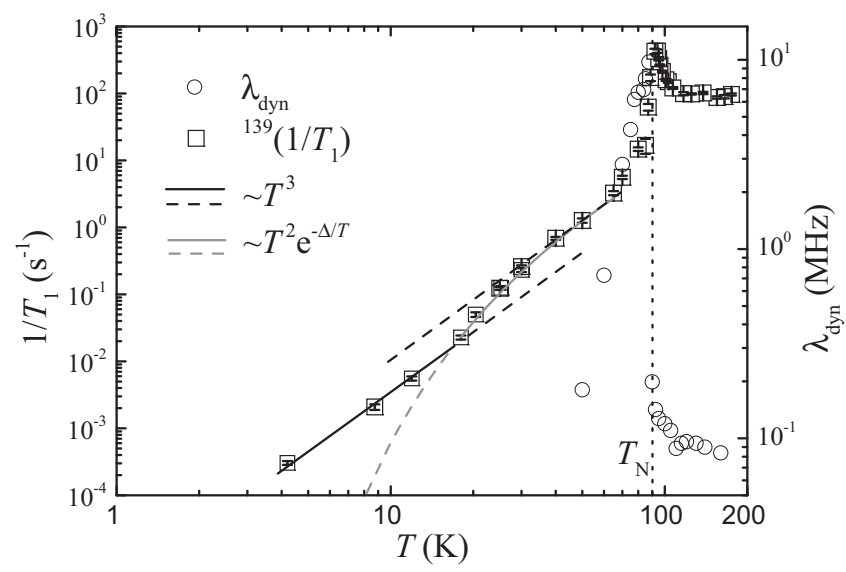

FIG. 10. The temperature dependence of ${ }^{139}\left(1 / T_{1}\right)$ is shown as open squares. The upper black dashed line shows the asymptotic $T^{3}$ power law for $T \gg \Delta$. The gray solid line and gray dashed line represent the model of activated two-magnon processes. The lower black lines show a $T^{3}$ power law behavior which is hidden for $T>20 \mathrm{~K}$. Open circles show dynamic relaxation rates $\lambda_{\text {dyn }}$ of the muon-spin polarization.

swept spectra for both ${ }^{139} \mathrm{La}$ sites. For site 1 also a good quantitative agreement is found (37\% difference between experimental and calculated hyperfine coupling). For site 2 the experimental value $A_{2, \perp}^{\prime}$ is approximately four times larger than the calculated $A_{2, \perp}$. This shows a limitation of the dipole approximation which does not take into account additional contributions of transferred hyperfine coupling.

The ${ }^{139} \mathrm{La}$ spin-lattice relaxation rate ${ }^{139}\left(1 / T_{1}\right)$ was measured by conventional saturation recovery method, exciting the central transition line at $44.0 \mathrm{MHz}$ and $7.297 \mathrm{~T}$ (marked by the vertical dash-dotted line in Fig. 6). Recovery curves are fitted by

$$
\begin{aligned}
1-\frac{M(t)}{M(0)}= & \frac{1}{84} \exp \left(-t / T_{1}\right)+\frac{3}{44} \exp \left(-6 t / T_{1}\right) \\
& +\frac{75}{364} \exp \left(-15 t / T_{1}\right)+\frac{1225}{1716} \exp \left(-28 t / T_{1}\right),
\end{aligned}
$$

taking into account magnetic transition probabilities $1 / T_{1}$ for a relaxation of a spin $I=7 / 2$ nuclei central transition. $M(t)$ is the value of the nuclear magnetization at a time $t$ after the saturation pulse, and $M(0)$ the equilibrium magnetization.

Figure 10 shows the ${ }^{139}\left(1 / T_{1}\right)$ vs $T$ plot together with the dynamic relaxation rate of the muon-spin polarization. For $T>T_{\mathrm{N}},{ }^{139}\left(1 / T_{1}\right)$ monitors the slowing down of magnetic fluctuations while approaching $T_{\mathrm{N}}$ with respect to equivalent ${ }^{139} \mathrm{La}$ sites. For $T<T_{\mathrm{N}}$ the experiments at $7.297 \mathrm{~T}$ mainly probe site 1 , and the spin-lattice relaxation is strongly reduced within an interval of $5 \mathrm{~K}$. For $T<65 \mathrm{~K}$ we find ${ }^{139}\left(1 / T_{1}\right) \propto T^{3}$ close to a power law. In the ordered state of AFM insulators the relaxation of nuclear spins in the presence of a gap $k_{B} \Delta$ in the magnetic excitation spectrum is mainly driven by Raman processes [24]. For $T \gg \Delta$ a $T^{3}$ behavior of the spin-lattice relaxation rate is expected for a dominating two-magnon process, while a three-magnon process will result in a $T^{5}$ power law [25]. The deviations of ${ }^{139}\left(1 / T_{1}\right)$ from the $T^{3}$ law towards lower temperatures indicates the crossover to the regime $T \ll \Delta$, where the thermal activation of gapped excitations will lead to ${ }^{139}\left(1 / T_{1}\right) \propto T^{2} e^{-\Delta / T}$. The gray line in Fig. 10 refers to $\Delta=55 \mathrm{~K}$, which is close to the observed gap in Ref. [19]. In the low-temperature regime the relaxation due to the gapped excitations is rapidly suppressed. For $T<20 \mathrm{~K}$ it is dominated by a process which again reveals a power law ${ }^{139}\left(1 / T_{1}\right) \propto T^{3}$.

\section{ZERO FIELD $\mu$ SR EXPERIMENTS}

In zero field $\mu \mathrm{SR}$ experiments the time dependent polarization $P(t)$ of an initially polarized muon-spin ensemble is obtained. After implantation, the positive muons thermalize very fast and come to rest at interstitial lattice sites. The muon decays into two neutrinos and a positron which is detected. Because the direction of the positron emission is favored along the direction of the muon spin at the moment of the decay, an ensemble average of several million decays provides $P(t)$. Representative spectra on powder samples of $\mathrm{La}_{2} \mathrm{O}_{2} \mathrm{Fe}_{2} \mathrm{OSe}_{2}$ at selected temperatures are shown in Fig. 11.

For $T>T_{\mathrm{N}}$ the polarization $P(t)$ is described by an exponential relaxation with two fractions $P_{\mathrm{dyn}}+P_{\mathrm{dyn}}^{\prime}=$ $100 \%$ [26]:

$$
P(t)=P_{\mathrm{dyn}} e^{-\lambda_{\mathrm{dyn}} t}+P_{\mathrm{dyn}}^{\prime} e^{-\lambda_{\mathrm{dyn}}^{\prime} t} .
$$

$\lambda_{\text {dyn }}$ and $\lambda_{\text {dyn }}^{\prime}$ denote relaxation rates of the time dependent muon-spin polarization. For $T>120 \mathrm{~K}$ we find $P_{\mathrm{dyn}}=$ $100 \%$, indicating magnetically equivalent muon sites in the paramagnetic regime.

In accordance with the onset temperature of the $V_{z z}$ reduction and the corresponding anomaly of the thermal expansion we identify a second fraction $P_{\text {dyn }}^{\prime} \approx 7 \%$ below $T=120 \mathrm{~K}$. This fraction exhibits a rather strong relaxation $\lambda_{\text {dyn }}^{\prime}>1.7 \mathrm{MHz} \gg \lambda_{\text {dyn }}$. We attribute this fraction of the $\mu \mathrm{SR}$ signal to muons affected by a large hyperfine coupling constant which are therefore probably located at an interstitial site close to a magnetic Fe moment. This conclusion will later be

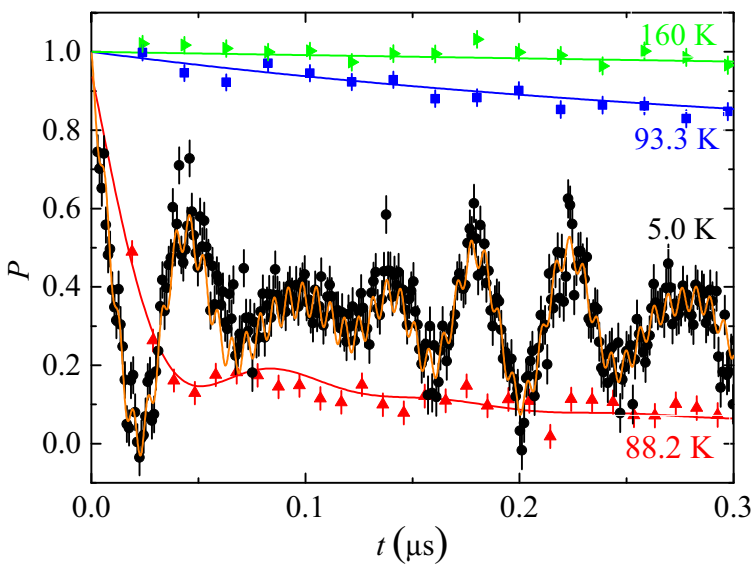

FIG. 11. (Color online) Representative zero field $\mu$ SR spectra and fits of $P(t)$ according to Eqs. (4) and (5). $T=93.3 \mathrm{~K}$ and $T=160 \mathrm{~K}:$ In the absence of long-range magnetic order $P(t)$ relaxes purely exponentially. $T=5 \mathrm{~K}: P(t)$ is fitted with a superposition of oscillating and nonoscillating fractions. $T=88.2 \mathrm{~K}$ : Oscillating and nonoscillating fractions are strongly affected by dynamic relaxation of the polarization. 


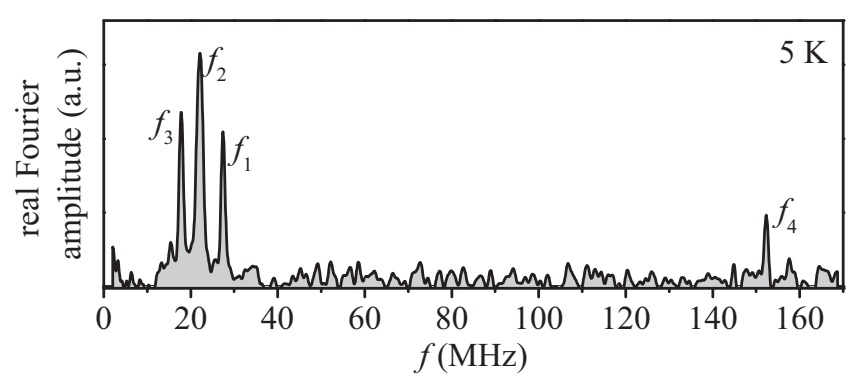

FIG. 12. The real Fourier amplitude of the muon-spin polarization measured at $T=5 \mathrm{~K}$ shows four well defined muon-spin precession frequencies.

reinforced by the observation of an equivalent signal fraction probing a strong static internal magnetic field in the lowtemperature, long-range ordered magnetic phase (see below).

$\lambda_{\text {dyn }}$ is shown in Fig. 10 and monitors the slowing down of magnetic fluctuations while approaching $T_{\mathrm{N}}$ in the paramagnetic regime.

In the magnetically long-range ordered regime $T<T_{\mathrm{N}}$ internal fields at the muon sites develop and therefore spontaneous muon-spin precession is observed. Taking into account the powder average, the polarization is described by $2 / 3$ oscillating and $1 / 3$ nonoscillating fractions. A magnetic five-site model for the oscillating fraction is identified from a Fourier transformation of the polarization $P(t)$ (Fig. 12), which displays four well defined frequencies and a fast relaxing contribution. The nonoscillating part of the polarization is described by an effective two-site model, with $85 \%$ dynamically depolarizing and 15\% nondepolarizing fractions [26]:

$$
P(t)=\frac{2}{3} \sum_{i=0}^{4} P_{i} e^{-\lambda_{T_{i}}} \cos \left(2 \pi f_{i} t\right)+\frac{1}{3}(\underbrace{0.85 e^{-\lambda_{\mathrm{dyn}} t}}_{\text {dyn. }}+\underbrace{0.15}_{\text {stat. }}) .
$$

Here $\lambda_{T_{i}}$ describe the depolarization of the oscillating signal fractions. The fast depolarization of fraction $P_{0}$ with $\lambda_{T, 0}>$ $50 \mathrm{MHz}$ prohibits a determination of $f_{0}$. We find signal fractions $P_{0}=36.4 \%, P_{1}=12.2 \%, P_{2}=33.1 \%, P_{3}=10.6 \%$, and $P_{4}=7.7 \%$ at lowest measured temperature $T=5 \mathrm{~K}$ and fix them for the analysis of the magnetically ordered regime. The obtained precession frequencies $f_{1}, f_{2}, f_{3}$, and $f_{4}$ are shown in Fig. 8. In our analysis the ratios of the frequencies $f_{1}, f_{2}$, and $f_{3}$ are determined from the $5 \mathrm{~K}$ spectrum and fixed for the further analysis. The high frequency oscillation $f_{4}$ nicely scales with the order parameter determined from ${ }^{57} \mathrm{Fe}$ Mössbauer and ${ }^{139} \mathrm{La}$ NMR experiments within error bars. In correspondence to the presented analysis of the ${ }^{57} \mathrm{Fe}$ Mössbauer order parameter the frequencies $f_{1}, f_{2}$ and $f_{3}$ yield a consistent critical exponent $\beta=0.15(1)$ and transition temperature $T_{\mathrm{N}}=88.5(8) \mathrm{K}$.

Fraction $P_{4}$ is nearly equal to $P_{\mathrm{dyn}}^{\prime}$ and $f_{4} \gg f_{1}, f_{2}, f_{3}$ indicates strong hyperfine coupling due to a close vicinity to magnetic Fe moments. We identify $P_{4}$ and $P_{\text {dyn }}^{\prime}$ as stemming from crystallographically equivalent muon sites. Spontaneous precession frequencies due to internal magnetic fields probe the magnetic order parameter. We find $f_{4}$ proportional to $B_{\text {hyp }}$ deduced from Mössbauer spectroscopy and $B_{2, \perp}$ deduced from

${ }^{139}$ La NMR experiments (Fig. 8).

We interpret the multiplicity of precession frequencies $f_{0}-f_{3}$ as due to magnetically nonequivalent sites in the ordered regime. Compared to the magnetic twofold splitting of ${ }^{139} \mathrm{La}$ spectra, a reduced symmetry of interstitial muon sites will lead to a more complex splitting of muon-spin precession frequencies.

Below $T_{\mathrm{N}}$ the dynamic depolarization $P_{\mathrm{dyn}}$ is reduced strongly. Finally for $T \leqslant 50 \mathrm{~K}$ only small residual depolarization $\lambda_{\text {dyn }}<0.1 \mathrm{MHz}$ is fitted (not shown in Fig. 10). Here the relaxation of the different muon fractions successively drops below the sensitivity of the method near $50 \mathrm{~K}$.

\section{SUMMARY}

We have investigated magnetic order and spin dynamics in $\mathrm{La}_{2} \mathrm{O}_{2} \mathrm{Fe}_{2} \mathrm{OSe}_{2}$ by means of ${ }^{57} \mathrm{Fe}$ Mössbauer spectroscopy, ${ }^{139} \mathrm{La}$ NMR, and muon-spin relaxation. The analysis of the Mössbauer spectra puts the system close to related oxychalcogenide systems (Table I). The spectra reveal the presence of magnetically equivalent $\mathrm{Fe}$ moments with noncollinear alignment parallel to $\mathrm{O}-\mathrm{Fe}-\mathrm{O}$ chains in the ordered state. This is in contrast to the collinear order model concluded in a neutron spectroscopy study but consistent with former theoretical predictions. The transition temperature to magnetic order is $T_{\mathrm{N}}=$ $90.1 \mathrm{~K}$. The critical exponent of the magnetic order parameter $\beta=0.133$ is close to that of the $2 \mathrm{D}$ Ising universality class.

In NMR field-swept experiments we find two magnetically nonequivalent ${ }^{139} \mathrm{La}$ sites in the magnetically ordered state. This is shown to be consistent with only one model of noncollinear magnetic order discussed in the literature, i.e., the model shown in Fig. 9(e). This model was already suggested as one possible model for $\mathrm{Nd}_{2} \mathrm{O}_{2} \mathrm{Fe}_{2} \mathrm{OSe}_{2}$ and was recently found to describe magnetic order in $\mathrm{Sr}_{2} \mathrm{~F}_{2} \mathrm{Fe}_{2} \mathrm{OS}_{2}$.

The observation of four muon-spin precession frequencies and a fast depolarizing signal in the ordered state in $\mathrm{La}_{2} \mathrm{O}_{2} \mathrm{Fe}_{2} \mathrm{OSe}_{2}$ is interpreted in terms of two different muon sites and the complex model of magnetic order.

Dynamic depolarization of the muon-spin ensemble and ${ }^{139} \mathrm{La}$ spin-lattice relaxation reveal a slowing down of magnetic fluctuations while approaching the long-range ordered state. For $T<T_{\mathrm{N}}$ magnetic fluctuations are strongly suppressed. In the temperature range $20<T<65 \mathrm{~K},{ }^{139}\left(1 / T_{1}\right)$ data probes the crossover to the gapped regime $T<T_{\Delta} \approx 55 \mathrm{~K}$, consistent with inelastic neutron scattering data [19]. For $T<20 \mathrm{~K}$ the strong reduction of spin-lattice relaxation rate due to the excitation gap is finally dominated by another source of relaxation, again leading to a power law behavior ${ }^{139}\left(1 / T_{1}\right) \propto T^{3}$. This is indicative for the presence of low-energy magnetic excitations below the gap energy. In the magnetically ordered state of $\mathrm{La}_{2} \mathrm{O}_{2} \mathrm{Fe}_{2} \mathrm{OSe}_{2}$ a small residual dynamic depolarization hides the signature of the spin excitation band [19].

Due to the peculiar local in-plane coordination of $\mathrm{Fe}$ atoms by oxygen, the magnetism in $\mathrm{La}_{2} \mathrm{O}_{2} \mathrm{Fe}_{2} \mathrm{OSe}_{2}$ is more anisotropic than in $\mathrm{Fe}$ pnictides. In the crystal electric field the strongly localized $S=2$ high spin states of $\mathrm{Fe}^{2+}$ form two orthogonal 2D Ising-like magnetic sublattices along O-Fe-O bonds. From the observed magnetic order we conclude AFM interaction via oxygen $J_{\mathrm{nnn} 1}$ and FM interaction via selenium 
$J_{\mathrm{nnn} 2}$. $\mathrm{La}_{2} \mathrm{O}_{2} \mathrm{Fe}_{2} \mathrm{OSe}_{2}$ is the second compound along with $\mathrm{Sr}_{2} \mathrm{~F}_{2} \mathrm{Fe}_{2} \mathrm{OS}_{2}$ were this particular order is observed.

\section{ACKNOWLEDGMENTS}

This work has been financially supported by the Deutsche Forschungsgemeinschaft (DFG) through the priority programm SPP 1458 (Projects No. KL 1086/10-1 and No. BU $887 / 15-1$ ) and the Research Training Group GRK 1621. R.S. is thankful to DFG for financial support via Grant No. DFG SA 2426/1-1. This work was supported by Korea NRF Grants (No. 2012-046138 and No. 2012M7A1A2055645). Part of this work was performed at the Swiss Muon Source (Villigen, Switzerland).
[1] C. de la Cruz, Q. Huang, J. W. Lynn, J. Li, W. R. II, J. L. Zarestky, H. A. Mook, G. F. Chen, J. L. Luo, N. L. Wang et al., Nature (London) 453, 899 (2008).

[2] H.-H. Klauss, H. Luetkens, R. Klingeler, C. Hess, F. J. Litterst, M. Kraken, M. M. Korshunov, I. Eremin, S.-L. Drechsler, R. Khasanov et al., Phys. Rev. Lett. 101, 077005 (2008).

[3] M. Rotter, M. Tegel, D. Johrendt, I. Schellenberg, W. Hermes, and R. Pöttgen, Phys. Rev. B 78, 020503 (2008).

[4] I. I. Mazin, Nature (London) 464, 183 (2010).

[5] Q. Si, Nat. Phys. 5, 629 (2009).

[6] R. M. Fernandes, A. V. Chubukov, J. Knolle, I. Eremin, and J. Schmalian, Phys. Rev. B 85, 024534 (2012).

[7] N. Ni, E. Climent-Pascual, S. Jia, Q. Huang, and R. J. Cava, Phys. Rev. B 82, 214419 (2010).

[8] L. L. Zhao, S. Wu, J. K. Wang, J. P. Hodges, C. Broholm, and E. Morosan, Phys. Rev. B 87, 020406(R) (2013).

[9] Y. Fuwa, T. Endo, M. Wakeshima, Y. Hinatsu, and K. Ohoyama, J. Am. Chem. Soc. 132, 18020 (2010).

[10] J. M. Mayer, L. F. Schneemeyer, T. Siegrist, J. V. Waszczak, and B. Van Dover, Angew. Chem. Int. Ed. Engl. 31, 1645 (1992).

[11] H. Kabbour, E. Janod, B. Corraze, M. Danot, C. Lee, M.-H. Whangbo, and L. Cario, J. Am. Chem. Soc. 130, 8261 (2008).

[12] Y. Fuwa, M. Wakeshima, and Y. Hinatsu, J. Phys.: Condens. Matter 22, 346003 (2010).
[13] D. G. Free, N. D. Withers, P. J. Hickey, and J. S. O. Evans, Chem. Mater. 23, 1625 (2011).

[14] S. Landsgesell, E. Blumenröther, and K. Prokeš, J. Phys.: Condens. Matter 25, 086004 (2013).

[15] H. Lei, E. S. Bozin, A. Llobet, V. Ivanovski, V. Koteski, J. Belosevic-Cavor, B. Cekic, and C. Petrovic, Phys. Rev. B 86, 125122 (2012).

[16] J.-X. Zhu, R. Yu, H. Wang, L. L. Zhao, M. D. Jones, J. Dai, E. Abrahams, E. Morosan, M. Fang, and Q. Si, Phys. Rev. Lett. 104, 216405 (2010).

[17] G. Wang, M. Zhang, L. Zheng, and Z. Yang, Solid State Commun. 151, 1231 (2011).

[18] D. G. Free and J. S. O. Evans, Phys. Rev. B 81, 214433 (2010).

[19] E. E. McCabe, C. Stock, E. E. Rodriguez, A. S. Wills, J. W. Taylor, and J. S. O. Evans, Phys. Rev. B 89, 100402 (2014).

[20] H. M. Rietveld, J. Appl. Crystallogr. 2, 65 (1969).

[21] T. Roisnel and J. Rodriguez-Carvajal, Mater. Sci. Forum 378381, 118 (2001).

[22] A. Suter and B. M. Wojek, Phys. Procedia 30, 69 (2012).

[23] G. Shirane, D. E. Cox, and S. L. Ruby, Phys. Rev. 125, 1158 (1962).

[24] T. Moriya, Prog. Theor. Phys. 16, 23 (1956).

[25] D. Beeman and P. Pincus, Phys. Rev. 166, 359 (1968).

[26] P. D. de Réotier and A. Yaouanc, J. Phys.: Condens. Matter 9 , 9113 (1997). 